\title{
Synthetic Cannabinoids: An Overview of The Clinical Pharmacology and Toxicology
}

\author{
*Benjamin DM \\ Department of Pharmaceutical Sciences, Northeastern University School of Pharmacy, USA \\ Submission: June 18, 2016; Published: August 16, 2016 \\ *Corresponding author: Benjamin DM, Department of Pharmaceutical Sciences, Northeastern University School of Pharmacy, USA
}

\section{Commentary}

Not only can cannabinoids be smoked or ingested, but cannabinoids are synthesized by the brain and are referred to as endogenous cannabinoids or endocannabinoids. The best known endocannabinoid is anandamide (AEA), the ethanolamine (amide) derivative of arachidonic acid. Another endocannabinoid, 2-arachidonoyl-glycerol (2-AG), also is synthesized in the brain. Arachidonic acid also is the precursor of prostaglandins and anandamide is biosynthesized in a similar "cascade-type" of "pattern."

Endocannabinoids play a major role during pre and postnatal development and also may be active in moderating fat metabolism and regulating blood vessels controlling blood pressure. However, in discussing endocannabinoid activity, the most important factor is its ability to bind to the $\mathrm{CB}_{1}$ receptor, which is responsible for the changes in mood and psychotic reactions following the smoking of marijuana or synthetic cannabinoids. Synthetic cannabinoids also up regulate the 5-HT-2A receptor (aka, the LSD receptor) and interact with dopamine receptors.

Synthetic cannabinoid $\mathrm{CB}_{1}$ receptors form homodimers with up to four subunits of other $\mathrm{CB}_{1}$ receptors and heterodimers with D2 dopamine receptors, mu opioid receptors, A2A adenosine receptors, and beta 2 adrenergic receptors. So, the SC user experiences dopamine, opioid, adenosine and beta- 2 stimulation in addition to a pure $\mathrm{CB}_{1}$ experience.

\section{Smoking THC (tetrahydrocannabinol) vs. Synthetic Cannabinoids}

Why is smoking synthetic cannabinoids more dangerous than smoking marijuana?: The word synthetic means to manufacture, prepare, or synthesize a chemical or drug, as opposed to naturally-occurring cannabinoids that are found in the marijuana plant, e.g., Cannabis Sativa. Currently, if you look at the structure of JWH-018, a prototype SC you will see that chemically, it is an indole. THC has no such indole structure, but LSD and DMT (di-methyltryptamine) do. So does serotonin, which also is known as 5-HT, which is an acronym for 5-hydroxytryptamine. The point of all this is to let you know that "Synthetic Cannabinoids" is a misleading name from a chemical perspective, since with the exception of the synthetic cannabinoids modeled after THC, (e.g., HU-210) all the synthetic cannabinoids possess vastly different chemical structures (mostly indoles). The reason these drugs are called cannabinoids is because they bind to the $\mathrm{CB}_{1}$ receptor and produce a number of psychopharmacologic and physiologic effects also produced by THC. So the relationship is that of similar pharmacologic effects on the body, not chemical similarity. In fact, the synthetic cannabinoids bind much more strongly to the $\mathrm{CB}_{1}$ receptor than $\mathrm{THC}$ and produce greatly intensified psychopharmacologic and physiologic effects resulting in seizures, heart attacks, panic attacks, and acute psychotic episodes.

Another reason smoking synthetic cannabinoids is more dangerous than smoking cannabis (marijuana) is because there are so many different synthetic cannabinoids, one may never be sure what they are smoking, and the concentrations and potencies of these agents (in Spice, for example) differ from batch to batch. For example an individual may purchase a bag of "Spice" but the contents of the bag will differ from other bags also called "Spice." In order to give the user the appearance of smoking marijuana, a synthetic cannabinoid is sprayed onto some type of vegetable matter, which serves as a burnable matrix which provides a base for the inhalation of the synthetic cannabinoid. No-one knows what the vegetable matter is, poisonous or safe, and even if "safe" what if you are allergic to that plant. You could have a "bee sting" like anaphylactic reaction and die on the spot!

The names or designations of the synthetic cannabinoids frequently have been derived from their discoverer or manufacturer. HU-210 is named for Hebrew University, where it was synthesized by Rafael Mechoulam in the 1980s. The CP compounds, or cyclohexylphenols, were developed by Pfizer pharmaceutical company as pain relievers in the late 1970 s and named CP for Charles Pfizer, with CP-47, 497 representing the prototypical drug. The class of aminoalkylindoles, the most prevalent type of synthetic cannabinoids, frequently bears the 
initials JWH, representing Clemson University Professor, John W. Huffmann, who first developed the JWH series in the late 1990s. This series originally included JWH-018, JWH-073, and JWH-200. Another class of synthetic cannabinoids, the phenylacetyliIndoles, often bears the designation RCS, which stands for Research Chemical Suppliers, of which RCS-8 is a prototypical example. Other synthetic cannabinoids include the benzoylindoles, which may bear a designation beginning with AM, which stands for Alexandros Makryannis, a synthetic organic chemist. Typical synthetic cannabinoids from this series include AM-694, AM-2201 and AM-1221. Agents designated as "Win" such as Win-55,212-2, an aminoalkylindole, were developed by Winthrop Labs., which used to be known as Sterling-Winthrop Pharmaceutical Company. Winthrop went on to synthesize more than 100 aminoalkylindole derivatives.

Thus it can be seen, that when an individual obtains a synthetic cannabinoid like Spice to smoke for recreational purposes, he or she may have no idea which chemical they are smoking, how concentrated it is, whether or not it has been purified to remove toxic contaminants, and what the dose is to obtain a pleasant marijuana-like experience, as opposed to a toxic, life-threatening adverse reaction (This assumes one can titrate the dose of SC and separate the euphoric and analgesic effects from the acute psychotic and psychotomimetic effects). Moreover, SCs upregulate the $5-\mathrm{HT}_{2 \mathrm{~A}}$ receptors which produces an array of severe psychopharmacologic effects usually seen in psychoses or schizophrenia, and usually not seen with THC.

\section{The Pharmacology and Toxicology of Synthetic Cannabinoids}

How THC and synthetic cannabinoids work in the brain: Neurologic signals in the brain are mediated through nerve pathways or tracts. Nerve pathways are a divergent or convergent path of nerve cells, or neurons, which lead from one area of the brain to another. Each neuron is capable of transmitting a signal to an adjacent neuron, even though neurons do not actually touch one another. Instead, neurons end just short of the next succeeding neuron leaving a gap or cleft between contiguous neurons. This gap is called the synaptic cleft or synapse. Neurons typically transmit signals to one another by releasing neurotransmitters just prior to the synapse (pre-synaptic neuron) and allowing the neurotransmitter to diffuse across the synaptic cleft and stimulate receptors located on the post-synaptic neuron. The binding of the neurotransmitter to the post-synaptic neuron initiates a response in that neuron. In the case of endocannabinoids, there is a feedback mechanism that permits release of endocannabinoids from presynaptic neuron membranes, where they are synthesized. The synthesis and release of the neurotransmitter in the pre-synaptic neuron membrane differs from the classical cholinergic neuron which stores acetylcholine in vesicles in the pre-synaptic neuron and releases the neurotransmitter when stimulated. Moreover, cannabinoid do not always act alone. Animal studies indicate that selective $\mathrm{CB}_{2}$ agonists up regulate $5-\mathrm{HT}_{2 \mathrm{~A}}$ and dopamine D2 receptors in the mouse brain, enhancing the interaction between $5-\mathrm{HT}_{2 \mathrm{~A}}$ and dopamine D2 receptors in the prefrontal cortex. Thus a CNS response to $\mathrm{SCs}$ involves $\mathrm{CB}_{1}$ receptors, $\mathrm{CB}_{2}$ receptors, up regulation of $5-\mathrm{HT}_{2 \mathrm{~A}}$ and dopamine $\mathrm{D} 2$ receptors in the prefrontal cortex.

Neural tracts can regulate motor functions such as walking, talking, moving your arms, scratching your head, or any other function in the body requiring the use of muscles. Neural tracts also can regulate sensory phenomena in the body such as touch, taste, vision, hearing, or pain. Neural tracts also are involved in the perception of emotions like happiness or sadness, and also can be involved in the extremes of happiness or sadness, such as euphoria (feeling high) or depression and anxiety. Many neuroscientists believe that research into the types of neurons and neurotransmitters involved with the downstream activity of $\mathrm{CB}_{1}$ receptors will lead to an understanding of the pathogenesis of such mental health disorders as depression, schizophrenia, and psychoses.

cannabinoid receptors: THC (tetrahydrocannabinol) and synthetic cannabinoids act by binding to specific cannabinoid receptors located on the neurons in various areas of the cortex and hippocampus. Receptors act like "light switches" ... when the drug molecule binds to the receptor, the switch is thrown and the neuron responds in an "all or none" manner, either increasing in firing or ceasing to function. In order to get a more intense response, more receptors have to be activated. The more receptors that are activated and the greater the affinity of the drug molecule for the receptor, the more intense the response.

THC (tetrahydrocannabinol) and synthetic cannabinoids are all Central Nervous System (CNS) depressants. In this context, a depressant means a drug that slows down or depresses the brain and slows you down, diminishes your cognitive functions, clouds your thinking, causes sleepiness, which in the case of synthetic cannabinoids, can progress to stupor, lack of consciousness, catatonia, and coma. THC (tetrahydrocannabinol) and synthetic cannabinoids act by binding to specific cannabinoid receptors located on the neurons in various areas of the cortex and hippocampus.

In the brain, many cannabinoid receptors are located in the frontal cortex, and in peripheral sites like mast cells, immune messenger cells, and vascular smooth muscle. There are 2 major types of cannabinoid receptors in the body, the $\mathrm{CB}_{1}$ and $\mathrm{CB}_{2}$ receptors. There also appears to be at least one or 2 other cannabinoids receptors but they have not yet been fully characterized. The $\mathrm{CB}_{1}$ receptor is located mainly in the cortex, but also can be found in the lungs, liver and kidneys. The $\mathrm{CB}_{2}$ receptors are found mainly in the immune system and the bloodforming cells. However, new locations in the CNS have been identified and additional effects are being discovered relating to the etiology of depression, schizophrenia, and other psychiatric disorders. Animal studies indicate that selective $\mathrm{CB}_{2}$ agonists up 
regulate $5-\mathrm{HT}_{2 \mathrm{~A}}$ and dopamine $\mathrm{D} 2$ receptors in the mouse brain, enhancing the interaction between $5-\mathrm{HT}_{2 \mathrm{~A}}$ and dopamine $\mathrm{D} 2$ receptors in the prefrontal cortex. The $\mathrm{CB}_{1}$ receptor is of great importance in cannabinoid pharmacology, since it is this receptor which mediates the psychopharmacologic effects of THC and synthetic cannabinoids.

The EMCDDA defines THC as a partial agonist for $\mathrm{CB}_{1}$ receptors (the $\mathrm{Ki}$ is very high at $10.2 \mathrm{~nm}$ ) in comparison to HU210, a structurally-modified THC with a Ki of $0.06 \mathrm{~nm}$, a 170 fold difference. Since neurons respond in an "all or none manner" the more neurons firing, the greater the magnitude of the response. With psychoactive drugs, occupation of a receptor, and sometimes the rate of binding to and unbinding from a receptor (occupancy rate) are related to the magnitude of the receptor's response.

When you look at the schematic structure of the $\mathrm{CB}_{1}$ and $\mathrm{CB}_{2}$ receptors, you may think that the configuration of the receptors looks something like a lock into which a key might fit. This is exactly how receptors work. The receptor acts as a lock, and drug molecules act as keys that can bind to the receptor (fit into the lock), stimulate the receptor to respond and produce a physiologic response in the body. In the case of THC and synthetic cannabinoids, these drug molecules bind to the cannabinoid receptors with various degrees of affinity (tightness of binding) and those drug molecules which bind with the greatest affinity, produce the most intense effect.

\section{Conclusion}

In closing, one of the reasons I became interested in the pharmacology and toxicology of SCs was the high number of adverse CNS effects and psychotic reactions reported, in contrast to the use of cannabis (THC). The data reported in this overview summarize the research findings related to the $\mathrm{CB}_{1}$ receptors and their interactions with the $5-\mathrm{HT}_{2 \mathrm{~A}}$ receptor (aka, the $\mathrm{LSD}$ receptor) and with the dopamine D2 receptors. it is also worthwhile to restate that THC, the prototypical cannabinoid agonist, is only a partial agonist for $\mathrm{CB}_{1}$ receptors (the Ki is very high at $10.2 \mathrm{~nm}$ ) in comparison to HU-210, a structurally-modified THC with a $\mathrm{Ki}$ of $0.06 \mathrm{~nm}$ ) while many SCs have much lower Ki's, bind to $\mathrm{CB}_{1}$ receptors with much greater affinity, and produce far more intense psychotomimetic effects than THC. These binding differences and the lack of CBD (cannabadiol) to modify the effect of the SC are the principal pharmacologic reasons why SCs are so much more toxic to the user than THC. 